Research Article

\title{
Ethyl Acetate Fraction from Hedyotis diffusa plus Scutellaria barbata Exerts Anti-Breast Cancer Effect via miR-200c-PDE7B/PD-L1-AKT/MAPK Axis
}

\author{
Yue Yang, ${ }^{1}$ Ting Fang $\mathbb{D},{ }^{1,2}$ Yi-Lan Cao, ${ }^{3}$ Ya-Xin Lv, ${ }^{1}$ Qing-Qi Chang, \\ and Dan-Dan Zhang $\mathbb{B}^{1}$ \\ ${ }^{1}$ Institute of Interdisciplinary Integrative Medicine Research, Shanghai University of Traditional Chinese Medicine, \\ Shanghai 201203, China \\ ${ }^{2}$ School of Pharmacy, Fujian University of Traditional Chinese Medicine, Fujian 350122, China \\ ${ }^{3}$ School of Pharmacy, Shanghai University of Traditional Chinese Medicine, Shanghai 201203, China
}

Correspondence should be addressed to Dan-Dan Zhang; izhangdd@126.com

Received 5 November 2019; Accepted 13 July 2020; Published 19 August 2020

Academic Editor: Salvatore Chirumbolo

Copyright (C) 2020 Yue Yang et al. This is an open access article distributed under the Creative Commons Attribution License, which permits unrestricted use, distribution, and reproduction in any medium, provided the original work is properly cited.

Background. Hedyotis diffusa (HD) Willd. and Scutellaria barbata (SB) D. Don in different ratios have been frequently used to treat various cancers in clinical Traditional Chinese Medicine prescriptions. However, the optimal ratio, active fraction, and molecular mechanisms associated with the anti-breast cancer role of this herbal couplet have not been elaborated. Methods. To screen out the optimal ratio of this herbal couplet, we compare aqueous extracts of HD, SB, or HD plus SB in different weight ratios (HS11, HS12, HS21) for their anticancer effects on murine breast cancer 4T1 cells in vitro and in vivo. EA11, the ethyl acetate fraction from HS11 (the aqueous extract of the couplet at an equal weight ratio), is further assessed for its antiproliferative effect as well as the antitumorigenic impact with the aid of immunocompetent mice. Colony formation, flow cytometry, western blot, ELISA, and qRT-PCR are used to elucidate mechanisms underlying EA11-led effects. Results. HS11 presents the most potential suppression of $4 \mathrm{~T} 1$ cell proliferation and tumor growth among these aqueous extracts. The comparison results show that EA11 is more effective than HS11 in vitro and in vivo. EA11 inhibits colony formation and induces apoptosis in a concentration-dependent manner. EA11 reduces the protein expressions of PDE7B, PD-L1, $\beta$-catenin, and cyclin D1 while elevating the concentration of cellular cAMP and miR-200c expression in $4 \mathrm{~T} 1$ cells. Additionally, EA11 exerts its anticancer effect partially via the inactivation of MAPK and AKT signaling pathways. Conclusions. This study implicates that EA11 prevents breast tumor development by interfering with the miR-200c-PDE7B/PD-L1-AKT/MAPK axis. EA11 may represent a potential therapeutic candidate for breast cancer.

\section{Introduction}

Breast cancer is a common neoplasm globally with a high incidence in younger women nowadays [1]. Triple-negative breast cancer (TNBC) accounts for $15-20 \%$ of all kinds of breast cancer as the most challenging subtype to cure. TNBCs are characterized by absence of estrogen receptor (ER), progesterone receptor (PR), and human epidermal growth factor receptor 2 (HER2). Moreover, the late-stage of TNBC is often accompanied by metastasis to bone, liver, and brain. Although surgery, radiation, and chemotherapy may improve the survival of TNBC patients, the mortality rate of recurrent patients is still high. Therefore, a novel therapeutic strategy is desirable for TNBCs. Cancer immunotherapy has demonstrated promising outcomes in the treatment of melanoma and lung cancer [2]. The programmed death ligand-1 (PD-L1)/programmed cell death receptor (PD-1) pathway plays a crucial role in immunotherapy. Notably, the binding within PD-L1 in tumor and PD-1 on the cell surface of $\mathrm{T}$ cells to inhibit cytotoxic $\mathrm{T}$ cell responses leads to immune surveillance and tumor development $[3,4]$. Recently, immunological checkpoint inhibitors have shown the efficiency of various cancers [5]. Anti-PD-L1 antibodies including atezolizumab (MPDL3280A), durvalumab 
(MEDI4736), avelumab, and pembrolizumab have been approved by Food and Drug Administration for the treatment of melanoma, Hodgkin's lymphoma, urothelial carcinoma, metastatic head and neck squamous cell carcinoma, and lung cancer [6-9].

Cyclic nucleotide phosphodiesterases (PDEs) are a multigene enzyme family that catalyzes the hydrolysis of cAMP (cyclic adenosine monophosphate) and cGMP (cyclic guanosine monophosphate). Among them, cyclic nucleotide phosphodiesterase isoform 7B (PDE7B) can specifically hydrolyze cAMP and regulate the intracellular concentrations, signaling pathways, and downstream biological functions of cAMP [10]. The higher level of PDE7B occurs in most glioblastoma cases and harms survival. The interaction between glioblastoma and endothelial cells leads to elevated PDE7B expression, which promotes tumor growth and aggressiveness [11]. PDE7 inhibitors selectively induce apoptosis in chronic lymphocytic leukemia cells due to PDE7B overexpression [12]. Therefore, PDE7B may be a potential target for some cancers.

Most mammalian mRNAs can be regulated by microRNAs (small noncoding RNAs, miRNAs) primarily at the posttranscriptional level, leading to either transcriptional silencing or degradation. miRNAs are 18-22-nucleotide endogenous noncoding RNAs that affect tumorigenic processes including cell proliferation, apoptosis, differentiation, metastasis, and drug resistance. There are five members in the miR-200 family: miR-200a, miR-200b, miR-200c, miR141, and miR-429 [13]. miR-200c is a multifunctional miRNA capable of regulating cell migration, proliferation, drug resistance, stemness, and metabolism disorders [14]. Members of the miR-200 family can target PD-L1 and ZEB1 to suppress the exhaustion of $\mathrm{CD} 8+\mathrm{T}$ cells and tumor metastasis in lung cancer [15]. miR-200c depressed PD-L1 expression and restored CD8+ $\mathrm{T}$ cell function in HBVmediated hepatocellular carcinoma [16]. In the previous study, miR-200c regulated the overexpression of PDE7B in TNBC cells and were critical for TNBC cell proliferation and tumor development by modulating cellular cAMP concentration [17]. Current studies have shown that Hedyotis diffusa Willd. (HD), an annual herb of the Rubiaceae family, exhibited numerous pharmacological activities [18-21]. Pharmacological evidence revealed that Scutellaria barbata (SB) D. Don also displayed anticancer, anti-inflammation, and immunity-enhancing effects. The anticancer effects of $\mathrm{HD}$ and SB are most likely to be mediated through the inhibition of cell proliferation, induction of cell apoptosis, and regulation of immunity [22-25]. HD and SB were always used with dosage combination at $1: 2,2: 1$, or $1: 1$ in clinical practice. Hedyotis diffusa and Scutellaria barbata (HDSB) are the core drug pair for the treatment of breast cancer and bladder cancer by data mining from clinical drug databases $[26,27]$. Our previous study showed that ethyl acetate fraction from the aqueous extract of the HDSB at equal weight ratio (EA11) has the most potent anti-inflammatory effect [28]. Given the close relationship between chronic inflammation and tumor, the objective of this study is to clarify the optimal ratio and active fraction from HDSB and to define the related antitumor mechanisms.

\section{Materials and Methods}

2.1. Cell Line and Culture Medium. The murine triple-negative breast cancer cell line $4 \mathrm{~T} 1$ was purchased from the American Type Culture Collection (ATCC, Rockville, MD, USA). The cells were cultured in Dulbecco's Modified Eagle Medium (DMEM) (Gibco, California, USA) containing 10\% FBS (Gibco, California, USA) in a $5 \% \mathrm{CO}_{2}$ incubator (Thermo, Waltham, USA) at $37^{\circ} \mathrm{C}$ constant temperature.

2.2. Herbal Extract Preparation Procedure. These herbal aqueous extracts were prepared. First, the dried powders of $\mathrm{HD}$ and $\mathrm{SB}$ in different weight ratios or alone (HS11:450 mg each; HS21: HD-600 mg, SB-300 mg; HS12: HD-300 mg, SB$600 \mathrm{mg}$; HD : $900 \mathrm{mg}$; SB : $900 \mathrm{mg}$ ) were mixed and boiled for $2 \mathrm{H}$. These supernatants were collected, filtrated, and concentrated separately. These herbal extracts were stored at $-20^{\circ} \mathrm{C}$ for further use. The source and identification of HD and $\mathrm{SB}$, and the preparation and quality control of EA11 were described in the previous study [28].

2.3. Cell Viability Assay. Cell viability was measured using MTT assay [28].

2.4. Immunocompetent Mice Bearing $4 \mathrm{T1}$ Tumor. All procedures performed in animal studies were approved by the Institutional Animal Care and Use Committee of Shanghai University of Traditional Chinese Medicine. The Female $\mathrm{Balb} / \mathrm{c}$ mice (6 weeks old) were purchased from Shanghai SLAC Laboratory Animal Co., Ltd., and maintained in a pathogen-free environment. $4 \mathrm{~T} 1$ cells $(5 * 10 E 5 / 100 \mu \mathrm{l})$ were injected into second mammary fat pads of mice. One week after cell injection, mice were randomly assigned to the following groups (6 mice/group): Model (vehicle, ig), HD, SB, HS11, HS12, HS21 (25 g crude herb/kg/d, ig), and EA11 $(50 \mathrm{mg} / \mathrm{kg} / \mathrm{d}$, ig). Mice were sacrificed after two weeks. Tumors were stripped, weighed, snap-frozen in liquid nitrogen, and stored at $-80^{\circ} \mathrm{C}$ for subsequent experiments.

2.5. Colony Formation Assay. Cells were seeded in a 6-well plate for adherence overnight. The cells were treated with EA11 $(25,50 \mu \mathrm{g} / \mathrm{ml})$ for $24 \mathrm{~h}$ in serum-free DMEM and replaced with 10\% FBS DMEM for six days. Cells were fixed with methanol and stained with $0.5 \%$ crystal violet (Genview, New Jersey, USA), and then the number of colonies was calculated.

2.6. Flow Cytometry. Apoptosis assay was performed using the PE/7-AAD Apoptosis Detection Kit (BD Biosciences, San Diego, CA, USA) on FACSCalibur according to the instructions. ModFit LT 3.0 software was used to calculate the percentage of apoptotic cells.

2.7. Western Blotting Analysis. 4T1 cells were treated with/ without EA11 for $24 \mathrm{~h}$, and cell lysates were collected using RIPA buffer (Beyotime Technology, Jiangsu, China) and 
mixed with phosphatase inhibitor (Roche, Basel, Switzerland). The concentrations of protein were determined by BCA Protein Assay Kit (Beyotime Technology, Jiangsu, China). Proteins were separated using 4-12\% SDS-PAGE gels and transferred to PVDF membranes. The membranes were blocked and then incubated with the following antibodies: PD-L1 (Proteintech, 17952-1-AP, $1: 1000)$, PDE7B (Abcam, ab170914, $1: 1000)$, $\beta$-catenin (CST, 9582, $1: 1000$ ), cyclin D1 (Abcam, ab134175, 1:5000), GAPDH (Proteintech, 10494-1-AP, $1: 10000$ ), JNK (CST, 9252, $1: 1000)$, p-JNK (CST, 9251, $1: 1000)$, ERK1/2 (CST, 9102, 1:1000), p-ERK1/2 (CST, 9101, 1:1000), p38/MAPK (CST, 9212, 1 : 1000), p-p38/MAPK (CST, 9211, 1:1000), p-AKT (CST, 4060, $1: 1000$ ), and AKT (CST, 9272, $1: 1000)$. Membranes were washed with PBS-Tween-20 buffer (PBS-T) and incubated with goat anti-rabbit IgG (Abcam, ab6721, $1: 10000)$. Blots were developed using ECL detection reagent (Millipore, Bedford, MA, USA). Protein bands were analyzed using the Tanon Imaging System (Tanon, Shanghai, China), and these band densities were quantified using the Tanon Program.

2.8. TaqMan ${ }^{\circledR}$ MicroRNA Real-Time RT-PCR Assays. Total RNA from each group was extracted by Trizol reagent (Invitrogen). hsa-miR-200c and the housekeeping gene U6 were determined by miScript II RT Kit and miScript SYBR Green PCR Kit (ABI, Foster City, CA, USA) according to the manufacturer's protocol. Real-time PCR amplifications were performed on ABI 7500 Fast Instrument. hsa-miR-200c expression was normalized to the endogenous control U6.

2.9. Determination of $c A M P$. The content of cAMP was determined using Cyclic AMP Select ELISA Kit (Cayman Chemical Company, Ann Arbor, MI) according to the manufacturer's protocol. 4T1 cells were treated with/without EA11 for $30 \mathrm{~min}$ before the analysis of cAMP.

2.10. Statistical Analysis. Statistical tests were performed using SPSS 20.0 (SPSS, Inc., Chicago, IL, USA). All results values are presented as mean \pm standard deviation (SD) from three independent experiments. Statistical analysis was performed by $t$-test or one-way ANOVA to determine the statistical significance.

\section{Results}

3.1. EA11 Exhibits a Potent Antitumor Effect In Vitro and In Vivo. Three weight ratios of $\mathrm{HD}$ and $\mathrm{SB}$ at $1: 1,2: 1$, or $1: 2$ are commonly used in clinical prescriptions to treat inflammatory diseases and cancers. We investigated the effect of aqueous extracts of HD, SB, HS11, HS12, HS21, and EA11 on 4T1 cell proliferation. MTT assay showed that HS11 exhibited the lowest $\mathrm{IC}_{50}$ at $226.93 \mu \mathrm{g} / \mathrm{mL}$ among all tested aqueous extracts. EA11, the ethyl acetate fraction from HS11, displayed the most potent inhibitory effect at $\mathrm{IC}_{50}$ of $30.28 \mu \mathrm{g} / \mathrm{mL}$ (Table 1). To further determine the impact of EA11 on tumor development, we injected 4T1 cells in female mice at second mammary fat pads. One week after cell injection, mice were administered with the vehicle control, HD, SB, HS11, HS12, HS21 (25 g crude drug/kg/d, equal to the clinical dosage), or EA11 (50 mg/kg/d) for two weeks. At the end of treatment, mice were sacrificed and tumors were excised. EA11 treatment group suppressed $66 \%$ of the tumor weight compared with the vehicle control group (Figure 1). These results showed that EA11 is the most potent in terms of suppressing tumor growth among all tested extracts.

3.2. EA11 Suppresses Colony Formation and Induces Apoptosis of 4 T1 Cells. To further characterize EA11's antitumorigenic effect, we assessed the ability of EA11 to block the colony formation assay. Results showed that EA11 reduced the ability of $4 \mathrm{~T} 1$ cells to form colonies in a concentrationdependent manner (Figure 2(a)). We also examined the effect of EA11 on apoptosis in 4T1 cells. The result of flow cytometry indicated that EA11 induced apoptosis in a concentration-dependent manner (Figure 2(b)).

3.3. EA11 Inhibits Protein Expressions of PD-L1, $P D E-7 B$, $\beta$-Catenin, and Cyclin D1 in 4T1 Cells. To define the molecular mechanisms associated with EA11-induced cell apoptosis, we analyzed the abundance of $\beta$-catenin and cyclin D1. As $\beta$-catenin and cyclin D1 are the critical components of the Wnt/ $\beta$-catenin pathway and are known to be essential for cell proliferation and survival, we observed that EA11 decreased the expressions of $\beta$-catenin and cyclin D1 (Figure 3), suggesting that Wnt signaling pathway was involved in EA11-led action.

Overexpression of PD-L1 and PDE7B was observed in TNBCs, so we further determined whether their expressions were affected by EA11 treatment. Western blot with specific antibodies showed that EA11 treatment downregulated the expressions of PD-L1 and PDE7B (Figure 3). These results suggested that EA11 suppressed tumorigenesis by the blockage of the expressions of PDE7B and PD-L1.

3.4. EA11 Elevates the Level of Cellular cAMP. Inducing cellular cAMP level by inhibiting PDE7B expression or activity has been reported to trigger apoptosis in chronic lymphocytic leukemia and breast cancer [10, 17]. The observation that EA11 downregulated PDE7B expression prompted us to measure cellular cAMP concentration in EA11-treated 4T1 cells. The level of cellular cAMP was significantly elevated by EA11 treatment (Figure 4), suggesting that EA11 may induce apoptosis partially by increasing cellular cAMP concentration through the suppression of PDE7B.

3.5. Effects of EA11 on AKT/MAPK Signaling Pathways. $\mathrm{PI} 3 \mathrm{~K} / \mathrm{AKT}$ and MAPK signaling pathways play vital roles in cell proliferation and survival. Both pathways are generally activated in TNBC cells $[29,30]$. Western blot showed that EA11 inhibited the degree of AKT phosphorylation while not affecting the amount of total AKT. Similarly, we observed that the levels of phosphorylation of ERK, JNK, and P38 were all 
TABLE 1: Screening extracts from HDSB and EA11 on cell proliferation $(n=3)$.

\begin{tabular}{|c|c|c|c|c|c|}
\hline Groups & Extracts/fractions & Dose $(\mu \mathrm{g} / \mathrm{ml})$ & Inhibition (\%) & $\mathrm{IC}_{50}(\mu \mathrm{g} / \mathrm{ml})$ & Yield (\%) \\
\hline \multirow[t]{2}{*}{ Control } & - & - & 0.00 & & \\
\hline & & 250 & 43.75 & 336.36 & 13.48 \\
\hline \multirow[t]{3}{*}{$\mathrm{HD}$} & Water extract & 500 & 53.15 & & \\
\hline & & 1000 & 81.68 & & \\
\hline & & 250 & 45.67 & 270.97 & 18.22 \\
\hline \multirow[t]{3}{*}{ SB } & Water extract & 500 & 59.51 & & \\
\hline & & 1000 & 58.87 & & \\
\hline & & 250 & 47.31 & 292.18 & 15.69 \\
\hline \multirow[t]{3}{*}{ HS12 } & Water extract & 500 & 56.53 & & \\
\hline & & 1000 & 74.56 & & \\
\hline & & 250 & 32.89 & 603.97 & 18.12 \\
\hline \multirow[t]{3}{*}{ HS21 } & Water extract & 500 & 43.83 & & \\
\hline & & 1000 & 59.22 & & \\
\hline & & 250 & 50.78 & 226.93 & 18.38 \\
\hline \multirow[t]{4}{*}{ HS11 } & Water extract & 500 & 68.52 & & \\
\hline & & 1000 & 80.69 & & \\
\hline & & 62.5 & 80.41 & 30.28 & 0.26 \\
\hline & & 125 & 91.71 & & \\
\hline \multirow[t]{3}{*}{ EA11 } & Ethyl acetate fraction & 250 & 94.58 & & \\
\hline & & 500 & 97.06 & & \\
\hline & & 1000 & 97.14 & & \\
\hline $\operatorname{Tax}$ & & $1(\mu \mathrm{M})$ & 70.50 & & \\
\hline
\end{tabular}

Cell viability of $4 \mathrm{~T} 1$ cells treated with extracts from HDSB and EA11 was determined by MTTassay. C: vehicle control; EA11: ethyl acetate fraction from HS11.

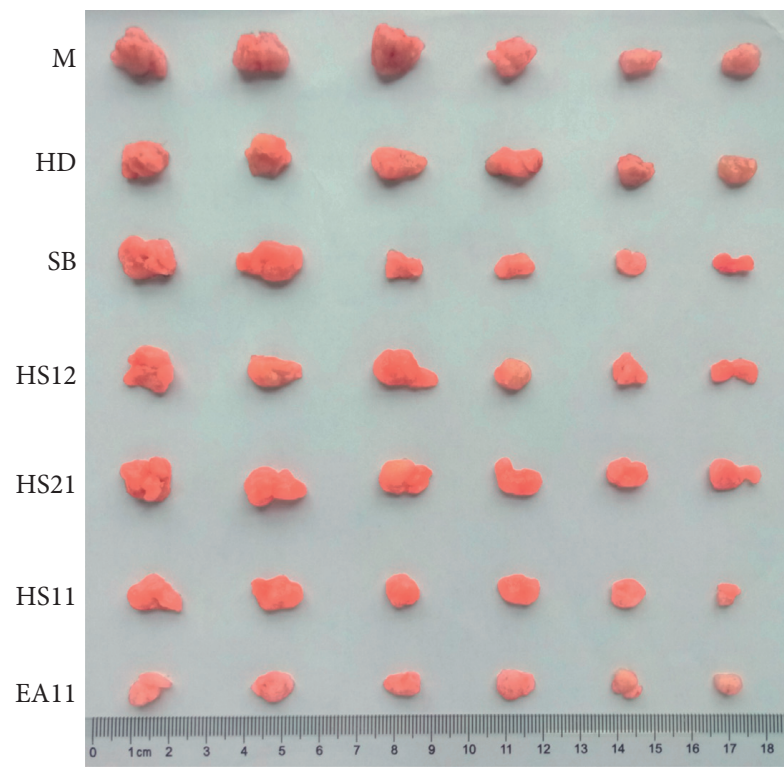

(a)

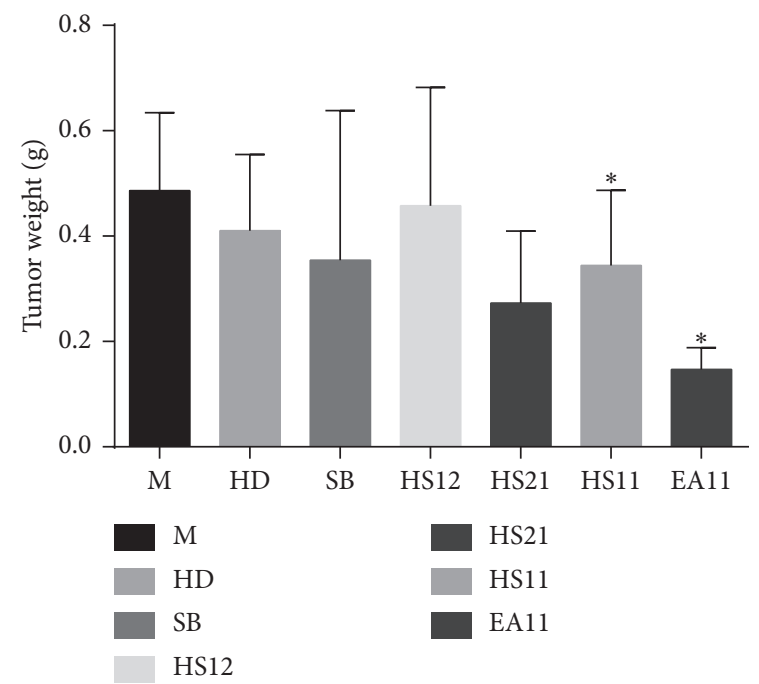

(b)

FIGURE 1: Effects on tumor growth of extracts from HDSB and EA11. M: model group; HD, SB, HS11, HS21, and HS11 at $25 \mathrm{~g}$ rude drug/kg/ d; EA11: ethyl acetate fraction from HS11 at $50 \mathrm{mg} / \mathrm{kg} / \mathrm{d}$; data are presented as means \pm standard deviation with three independent experiments. ${ }^{*} P<0.05$ vs. $M$ group.

reduced upon EA11 treatment (Figure 5). These results suggested that EA11 may also exert its antitumorigenic effect by partially inhibiting AKT/MAPK signaling pathways.

3.6. Effect of EA11 on miR-200c Expression. miRNAs directly or indirectly regulate AKT/MAPK signaling pathways. Our previous study showed that miR-200c-led inhibition of
PDE7B diminished AKT activity to regulate TNBC cell proliferation and tumor growth [17]. Thus, we investigated whether miR-200c was associated with EA11-induced inactivation of AKT signal pathway. After the treatment with EA11, we observed that the level of miR-200c was significantly increased in $4 \mathrm{~T} 1$ cells (Figure 6). These results indicated that EA11 induced miR-200c expression, leading to 


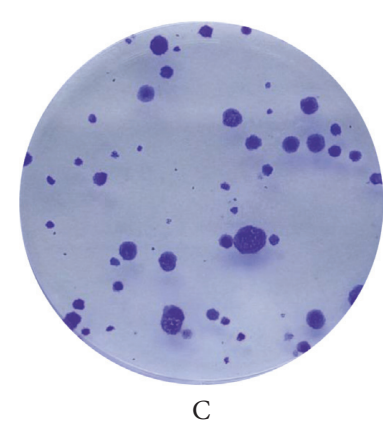

C

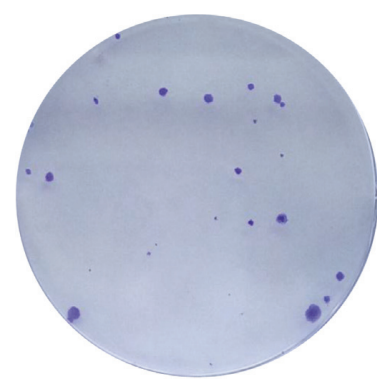

25

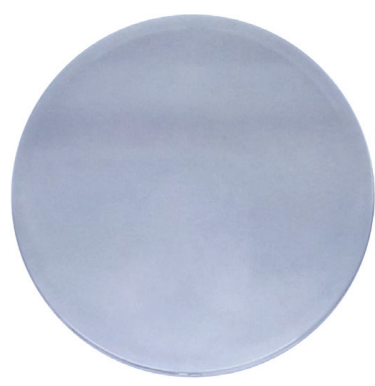

50

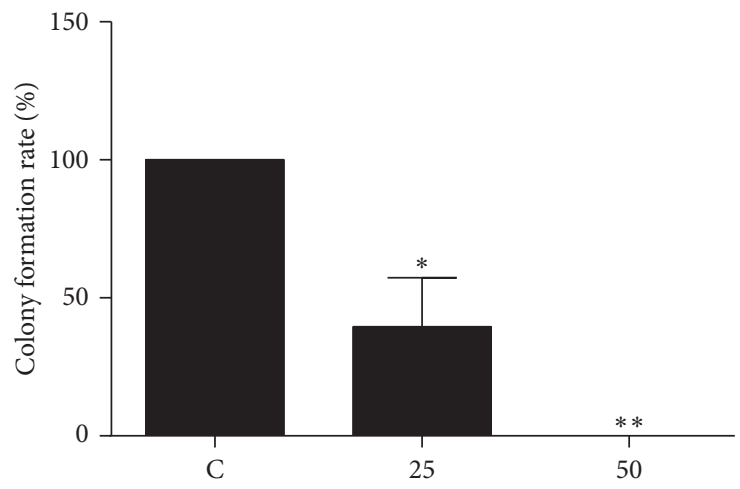

(a)
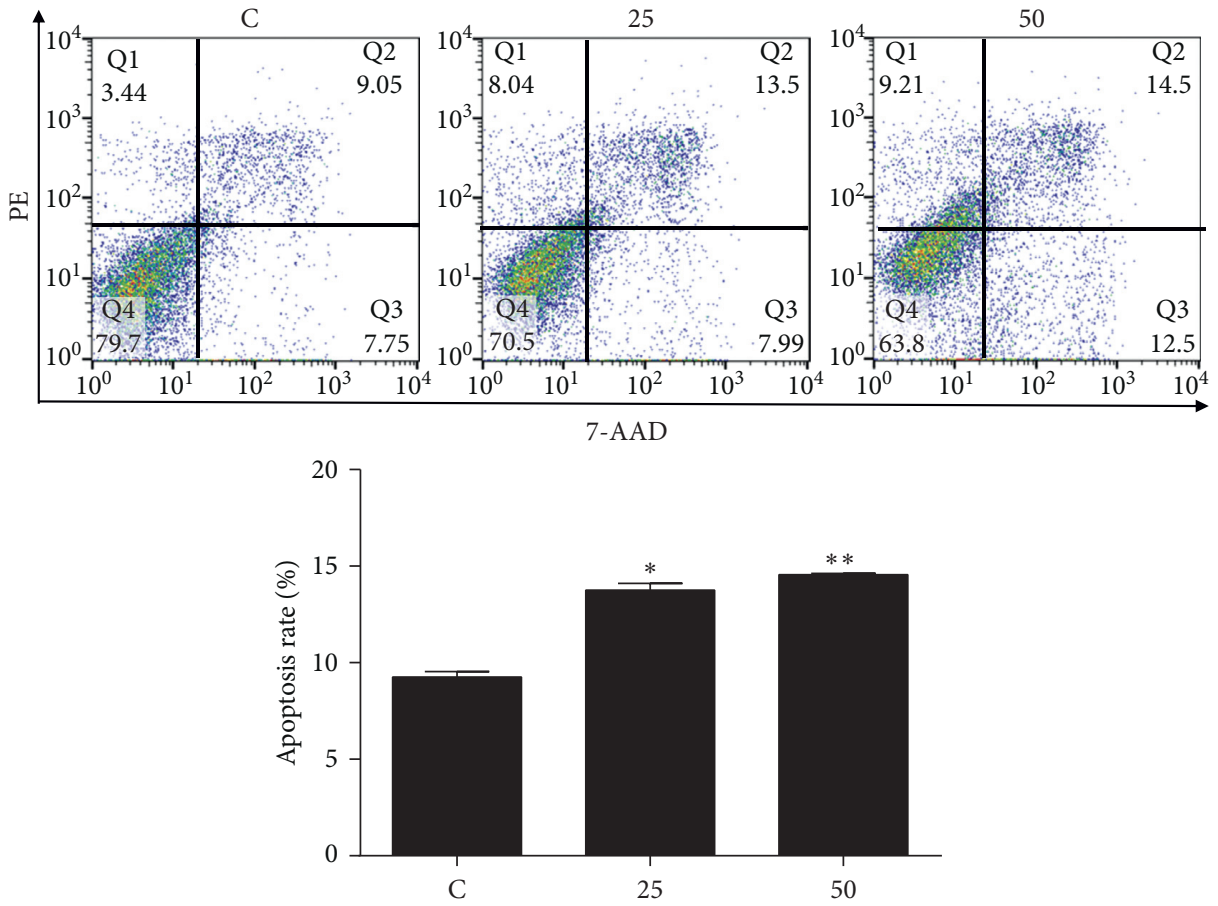

(b)

FIGURE 2: Colony formation inhibition and apoptosis induction of EA11. Following treatment of $4 \mathrm{~T} 1 \mathrm{cells}$ with $25 \mathrm{or} 50 \mu \mathrm{g} / \mathrm{ml}$ of EA11, (a) colony formation assay and (b) cell apoptosis assay were carried out. ${ }^{*} P<0.05,{ }^{* *} P<0.01$ vs. C group. 


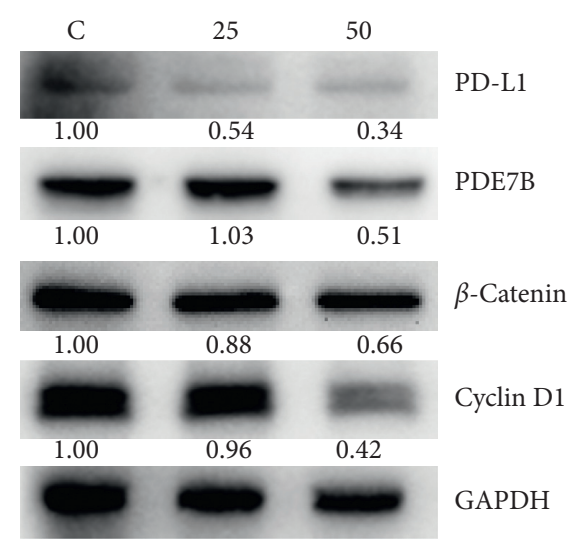

(a)

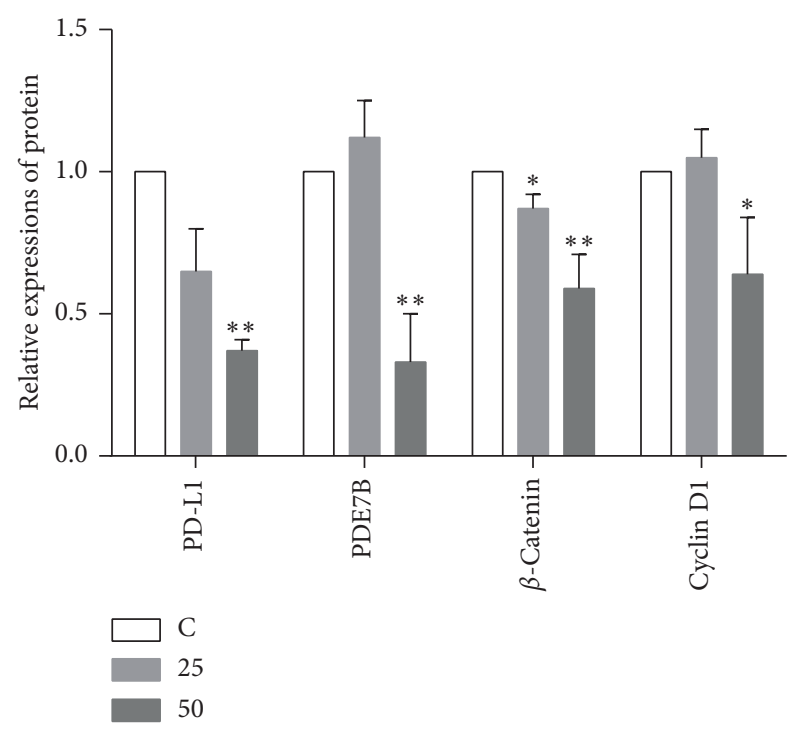

(b)

FIGURe 3: Analysis expressions of PDE7B, PD-L1, $\beta$-catenin and cyclin D1. Following the treatment of $4 \mathrm{~T} 1 \mathrm{cells}$ with $25 \mathrm{or} 50 \mu \mathrm{g} / \mathrm{ml}$ of EA11, PDE7B, PD-L1, $\beta$-catenin and cyclin D1, proteins were assessed by western blot. Values are presented as mean \pm SD from three experiments. ${ }^{*} P<0.05,{ }^{* *} P<0.01$ vs. C group.

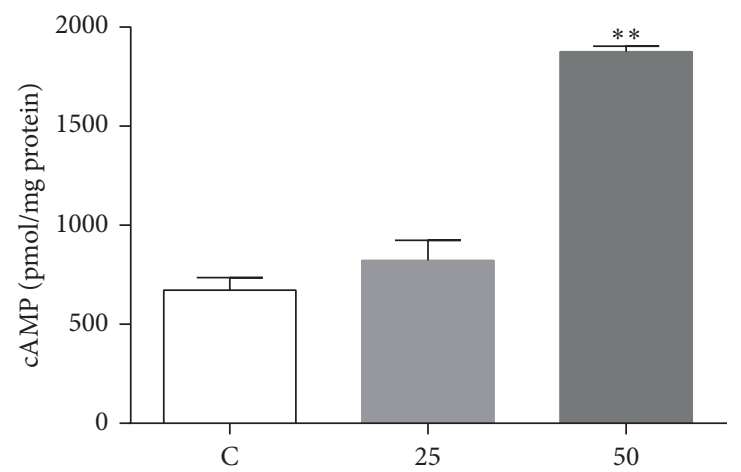

Figure 4: Determination of cAMP. Following the treatment of 4T1 cells with 25 or $50 \mu \mathrm{g} / \mathrm{ml}$ EA11 for 30 min, the level of cAMP in each group was assessed by ELISA. ${ }^{* *} P<0.01$ vs. C group.

the inhibition of PDE7B expression and the subsequent inactivation of AKT signaling pathways.

\section{Discussion}

It is believed that tumors are the accumulation of heat and toxicity according to Traditional Chinese Medicine (TCM) theory. Therefore, the principle of clearing the heat and removing the toxins is one of the promising strategies for the treatment of cancer. Hedyotis diffusa and Scutellaria barbata have been widely used to treat various cancer types, and there are three weight ratios of this herb pair in prescriptions. However, the optimal weight ratio of $\mathrm{HD}$ plus SB in breast cancer treatment is not evaluated. In this study, we showed that HD plus SB at an equal weight ratio (HS11) exhibited a more potent antitumor effect than the other two weight ratios. EA11, the ethyl acetate fraction from HS11, displayed a more substantial impact on $4 \mathrm{~T} 1$ cell proliferation and tumor growth than HS11 (Table 1). EA11 inhibited colony formation and induced cell apoptosis in a concentration-dependent manner (Figure 2). EA11 potently diminished the expressions of $\beta$-catenin and cyclin D1 (Figure 3); we reasoned the possibility that EA11 partially exerted its anticancer effect by interfering with Wnt signaling. MiR-200c regulates epithelial-mesenchymal transition (EMT) by blocking TGF- $\beta$, PI3K/AKT, Notch, VEGF, and NF- $\kappa$ B signaling pathways [14]. miR$200 \mathrm{c}$ inhibited the expression of PDE7B, leading to an increased level of cellular cAMP and subsequent AKT inactivation and growth inhibition in TNBC cells [17]. In this study, EA11 increased the level of miR-200c, inhibited the expression of target gene PDE7B (Figures 3-6), elevated the concentration of cellular CAMP, and diminished the AKT activity (Figure 5). EA11 inhibited 4T1 proliferation and growth by the miR-200c-PDE7B-cAMP-AKT pathway. 


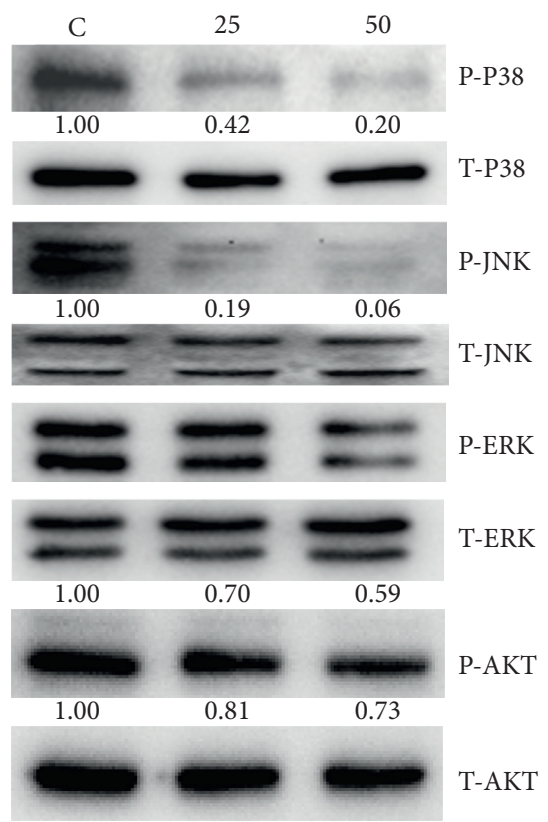

(a)

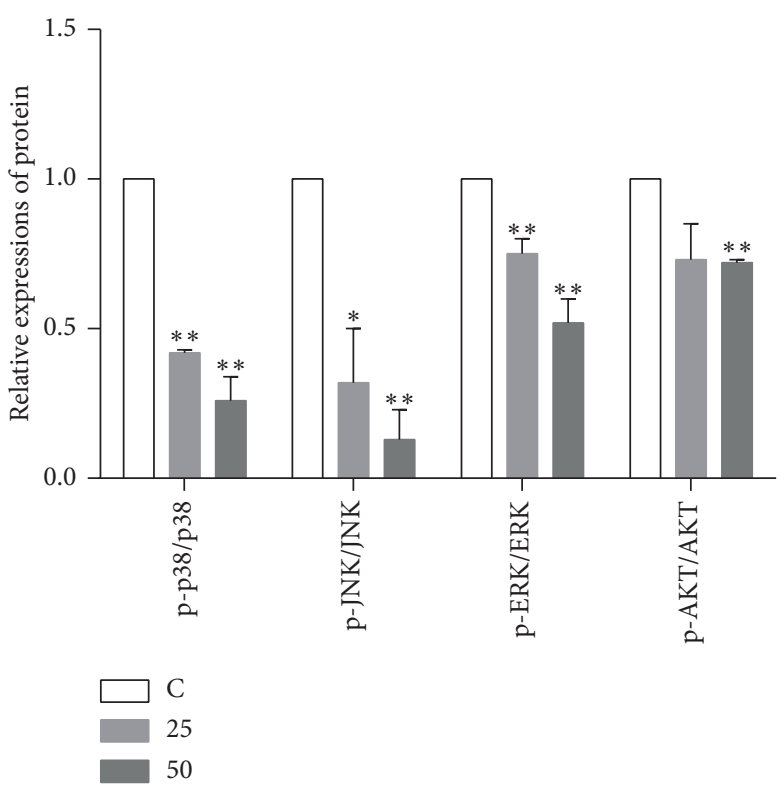

(b)

FIgURE 5: Effect of EA11 on the AKT and MAPK signaling pathways. p-ERK, ERK, p-JNK, JNK, p-p38, p38, p-AKT, and AKT protein expressions were assessed by western blot following treatment with 25 or $50 \mu \mathrm{g} / \mathrm{ml}$ of EA11. Band intensity was determined using an imaging system. Band expression levels were calculated relative to the intensity of total JNK/ERK/p38/AKT protein. ${ }^{*} P<0.05,{ }^{* *} P<0.01$ vs. C group.

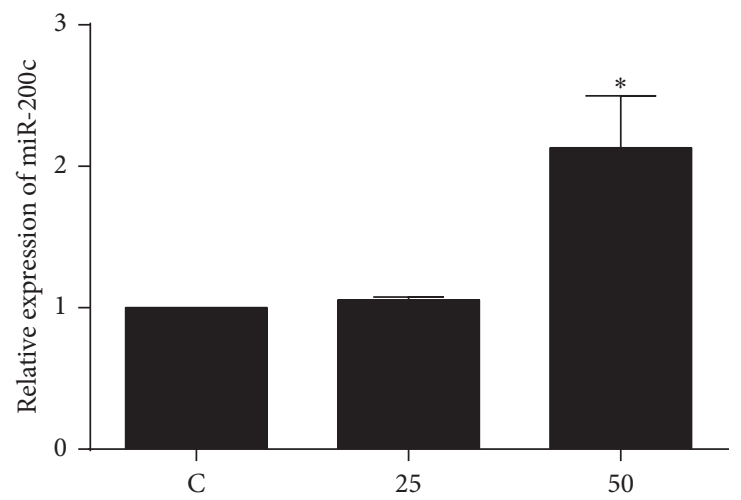

Figure 6: Effect of EA11 on miR-200c levels in murine 4T1 cells. $4 \mathrm{~T} 1$ cells were incubated with the treatment of $25 \mathrm{and} 50 \mu \mathrm{g} / \mathrm{ml}$ EA11 for $24 \mathrm{~h} ;{ }^{*} \mathrm{P}<0.05$ vs. C group.

Tumor cells can escape the surveillance from the body's immune system through a wide range of mechanisms including attraction of immunosuppressive cells, secretion of various cytokines and chemokines, and induction of immune checkpoint-mediated cosuppression signaling pathways. The expression of immunological checkpoint proteins in tumor cells results in the negative feedback of immune response and thereby promotes tumor development and metastasis [31]. Lack of miR-200c significantly increased the expressions of PD-L1 and MUC1 oncoprotein in acute myeloid leukemia, which showed that miR-200c was one of the critical negative regulators of PD-L1 [32]. Data analysis of 98 patients with $\mathrm{HBV}$-associated hepatocellular carcinoma revealed that PD-L1 was negatively correlated with
miR-200c [16] as well as that observed in clinical specimens of colon cancer [33]. In this study, EA11 robustly upregulated the expression of miR-200c to inhibit PD-L1 expression in $4 \mathrm{~T} 1$ cells.

The MAPKs pathway is responsible for cell survival, proliferation, and differentiation including ERK1/2, JNK/ SAPK, p38, and ERK5. Mutation-driven perturbations of MAPK pathways in breast tumors are linked to the negative regulation of immune response in breast cancer [34]. Combined Ras-MAPK pathway and PD-L1/PD-1 inhibition enhanced antitumor immune sensitivity in TNBC [35]. The data indicated that EA11 had inhibitory effects on phosphorylation of p38, JNK, and ERK/MAPK on 4 T1 cells. Thus, inactivation of MAPKs pathway may boost the immune 


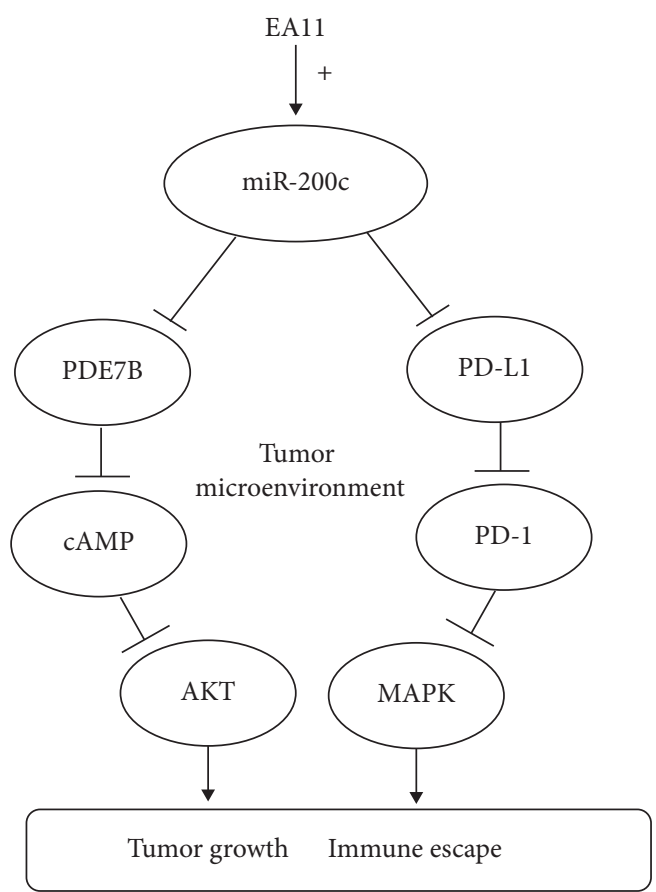

Figure 7: Potential molecular mechanisms by EA11. EA11 may induce miR-200c expression to regulate PDE7B and PD-L1 via AKT/MAPK signal pathways to suppress $4 \mathrm{~T} 1$ proliferation and growth.

response by regulation of PD-L1 (Figure 5). In conclusion, our study demonstrated that EA11 could suppress breast tumor development by interfering with the miR-200cPDE7B/PD-L1-AKT/MAPK axis (Figure 7). Based on these findings, EA11 can be developed as a chemopreventive agent for TNBC treatment.

\section{Data Availability}

The original data used to support the findings of this study are available from the corresponding author upon request.

\section{Conflicts of Interest}

The authors declare that there are no conflicts of interest regarding the publication of this paper.

\section{Authors' Contributions}

Yue Yang, Ting Fang, and Yi-Lan Cao contributed equally to this work.

\section{Acknowledgments}

This work was supported by the National Natural Science Foundation of China $(81773946,81573673,81001666)$ and Innovation Project for Undergraduates of Shanghai University of Traditional Chinese Medicine (2017SHUTCM041).

\section{References}

[1] Y. He, Z. Jiang, C. Chen, and X. Wang, "Classification of triple-negative breast cancers based on Immunogenomic profiling," Journal of Experimental \& Clinical Cancer Research, vol. 37, no. 1, p. 327, 2018.

[2] P. Sharma, "Biology and management of patients with triplenegative breast cancer," The Oncologist, vol. 21, no. 9, pp. 1050-1062, 2016.

[3] C. Blank, T. F. Gajewski, and A. Mackensen, "Interaction of PD-L1 on tumor cells with PD-1 on tumor-specific T cells as a mechanism of immune evasion: implications for tumor immunotherapy," Cancer Immunology, Immunotherapy, vol. 54, no. 4, pp. 307-314, 2005.

[4] M. E. Keir, M. J. Butte, G. J. Freeman, and A. H. Sharpe, "PD-1 and its ligands in tolerance and immunity," Annual Review of Immunology, vol. 26, no. 1, pp. 677-704, 2008.

[5] X. Meng, Y. Liu, J. Zhang, F. Teng, L. Xing, and J. Yu, "PD-1/ PD-L1 checkpoint blockades in non-small cell lung cancer: new development and challenges," Cancer Letters, vol. 405, pp. 29-37, 2017.

[6] K. Meredith, J. Chuk, T. Chang, and M. R. Theoret, "FDA approval summary: accelerated approval of pembrolizumab for second-line treatment of metastatic melanoma," Clinical Cancer Research, vol. 23, no. 19, pp. 5666-5670, 2017.

[7] Y. L. Kasamon, R. A. D. Claro, Y. Wang et al., "FDA approval summary: nivolumab for the treatment of relapsed or progressive classical hodgkin lymphoma," Oncologist, vol. 22, no. 5, pp. 585-591, 2017.

[8] Y. M. Ning, D. Suzman, V. E. Maher et al., "FDA approval summary: atezolizumab for the treatment of patients with progressive advanced urothelial carcinoma after platinumcontaining chemotherapy," The Oncologist, vol. 22, no. 6, pp. 743-749, 2017.

[9] E. Larkins, G. M. Blumenthal, W. Yuan et al., "FDA approval summary: pembrolizumab for the treatment of recurrent or metastatic head and neck squamous cell carcinoma with disease progression on or after platinum-containing chemotherapy," The Oncologist, vol. 22, no. 7, pp. 873-878, 2017. 
[10] F. Murray and P. A. Insel, "Targeting cAMP in chronic lymphocytic leukemia: a pathway-dependent approach for the treatment of leukemia and lymphoma," Expert opinion on therapeutic targets, vol. 17, no. 8, pp. 937-949, 2013.

[11] M. D. Brooks, E. Jackson, N. M. Warrington et al., "PDE7B is a novel, prognostically significant mediator of glioblastoma growth whose expression is regulated by endothelial cells," PLoS One, vol. 9, no. 9, Article ID e107397, 2014.

[12] L. Luo, F. Murray, A. Zahno et al., "Cyclic nucleotide phosphodiesterase profiling reveals increased expression of phosphodiesterase 7B in chronic lymphocytic leukemia," Proceedings of the National Academy of Sciences, vol. 105, no. 49, pp. 19532-19537, 2008.

[13] G.-L. Huang, J. Sun, Y. Lu et al., "MiR-200 family and cancer: from a meta-analysis view," Molecular Aspects of Medicine, vol. 70, pp. 57-71, 2019.

[14] M. Mutlu, U. Raza, Ö. Saatci, E. Eyüpoğlu, E. Yurdusev, and Ö. Şahin, "miR-200c: a versatile watchdog in cancer progression, EMT, and drug resistance," Journal of Molecular Medicine, vol. 94, no. 6, pp. 629-644, 2016.

[15] L. Chen, D. L. Gibbons, S. Goswami et al., "Metastasis is regulated via microRNA-200/ZEB1 axis control of tumour cell PD-L1 expression and intratumoral immunosuppression," Nature Communications, vol. 5, no. 1, p. 5241, 2014.

[16] C. Sun, P. Lan, Q. Han et al., "Oncofetal gene SALL4 reactivation by hepatitis B virus counteracts miR-200c in PD-L1induced T cell exhaustion," Nature Communications, vol. 9, no. 1, p. 1241, 2018.

[17] D.-D. Zhang, Y. Li, Y. Xu, J. Kim, and S. Huang, "Phosphodiesterase $7 \mathrm{~B} /$ microRNA-200c relationship regulates triple-negative breast cancer cell growth," Oncogene, vol. 38, no. 7, pp. 1106-1120, 2019.

[18] L. Lin, K. Cheng, Z. Xie et al., "Purification and characterization a polysaccharide from Hedyotis diffusa and its apoptosis inducing activity toward human lung cancer cell line A549," International Journal of Biological Macromolecules, vol. 122, pp. 64-71, 2019.

[19] Y.-J. Kuo, J.-S. Yang, C.-C. Lu, S.-Y. Chiang, J.-G. Lin, and J.-G. Chung, "Ethanol extract of Hedyotis diffusa willd upregulates G0/G1 phase arrest and induces apoptosis in human leukemia cells by modulating caspase cascade signaling and altering associated genes expression was assayed by cDNA microarray," Environmental Toxicology, vol. 30, no. 10, pp. 1162-1177, 2015.

[20] P. Zhang, B. Zhang, J. Gu, L. Hao, F. Hu, and C. Han, "The study of the effect of Hedyotis diffusa on the proliferation and the apoptosis of the cervical tumor in nude mouse model," Cell Biochemistry and Biophysics, vol. 72, no. 3, pp. 783-789, 2015.

[21] C. Sun, J. Yang, H.-B. Cheng et al., "2-Hydroxy-3-methylanthraquinone inhibits lung carcinoma cells through modulation of IL-6-induced JAK2/STAT3 pathway," Phytomedicine, vol. 61, Article ID 152848, 2019.

[22] P. Sun, D. Sun, and X. Wang, "Effects of Scutellaria barbata polysaccharide on the proliferation, apoptosis and EMT of human colon cancer HT29 cells," Carbohydrate Polymers, vol. 167, pp. 90-96, 2017.

[23] J. Lin, J. Feng, H. Yang et al., "Scutellaria barbata D. Don inhibits 5-fluorouracil resistance in colorectal cancer by regulating PI3K/AKT pathway," Oncology Reports, vol. 38, no. 4, pp. 2293-2300, 2017.

[24] Y. Jin, W. Chen, H. Yang et al., "Scutellaria barbata D. Don inhibits migration and invasion of colorectal cancer cells via suppression of PI3K/AKT and TGF- $\beta /$ Smad signaling pathways," Experimental and Therapeutic Medicine, vol. 14, no. 6, pp. 5527-5534, 2017.

[25] X. Kan, W. Zhang, R. You, Y. Niu, J. Guo, and J. Xue, "Scutellaria barbata D. Don extract inhibits the tumor growth through down-regulating of treg cells and manipulating Th1/ Th17 immune response in hepatoma H22-bearing mice," BMC Complementary and Alternative Medicine, vol. 17, no. 1, p. 41, 2017.

[26] Y.-C. Yeh, H.-Y. Chen, S.-H. Yang et al., "Hedyotis diffusa combined with Scutellaria barbata are the core treatment of Chinese herbal medicine used for breast cancer patients: a population-based study," Evidence-Based Complementary and Alternative Medicine, vol. 2014, Article ID 202378, 9 pages, 2014.

[27] L.-T. Pan, Y. Sheung, W.-P. Guo, Z.-B. Rong, and Z.-M. Cai, "Hedyotis diffusa plus Scutellaria barbata induce bladder cancer cell apoptosis by inhibiting akt signaling pathway through downregulating miR-155 expression," EvidenceBased Complementary and Alternative Medicine, vol. 2016, Article ID 9174903, 10 pages, 2016.

[28] Y. Xu, X.-X. Chen, Y.-X. Jiang, and D.-D. Zhang, "Ethyl acetate fraction from Hedyotis diffusa plus Scutellaria barbata exerts anti-inflammatory effects by regulating miR-155 expression and JNK signaling pathway," Evidence-Based Complementary and Alternative Medicine, vol. 201811 pages, Article ID 3593408, 2018.

[29] D. Massihnia, A. Galvano, D. Fanale et al., "Triple negative breast cancer: shedding light onto the role of pi3k/akt/mtor pathway," Oncotarget, vol. 7, no. 37, pp. 60712-60722, 2016.

[30] Y. Sun, W.-Z. Liu, T. Liu, X. Feng, N. Yang, and H.-F. Zhou, "Signaling pathway of MAPK/ERK in cell proliferation, differentiation, migration, senescence and apoptosis," Journal of Receptors and Signal Transduction, vol. 35, no. 6, pp. 600-604, 2015.

[31] A. M. Intlekofer and C. B. Thompson, "At the bench: preclinical rationale for CTLA-4 and PD-1 blockade as cancer immunotherapy," Journal of Leukocyte Biology, vol. 94, no. 1, pp. 25-39, 2013.

[32] A. R. Pyzer, D. Stroopinsky, J. Rosenblatt et al., "MUC1 inhibition leads to decrease in PD-L1 levels via upregulation of miRNAs," Leukemia, vol. 31, no. 12, pp. 2780-2790, 2017.

[33] C. Martinez-Ciarpaglini, S. Oltra, S. Roselló et al., "Low miR200c expression in tumor budding of invasive front predicts worse survival in patients with localized colon cancer and is related to PD-L1 overexpression," Modern Pathology, vol. 32, no. 2, pp. 306-313, 2019.

[34] W. Hendrick, I. Simeone, S. Anjum et al., "Identification of genetic determinants of breast cancer immune phenotypes by integrative genome-scale analysis," Oncoimmunology, vol. 6, no. 2, Article ID e1253654, 2017.

[35] S. Loi, S. Dushyanthen, P. A. Beavis et al., "RAS/MAPK activation is associated with reduced tumorinfiltrating lymphocytes in triple-negative breast cancer: therapeutic cooperation between MEK and PD-1/PD-L1 immune checkpoint inhibitors," Clinical Cancer Research, vol. 22, no. 6, pp. 1499-1509, 2016. 